\title{
Cognitive and metacognitive factors among alcohol-dependent patients during a residential rehabilitation program: a pilot study
}

This article was published in the following Dove Press journal:

Neuropsychiatric Disease and Treatment

\author{
Elisa Torselli' \\ Marcella Ottonello ${ }^{2}$ \\ Emilio Franceschina' \\ Emanuele Palagi' \\ Giorgio Bertolotti ${ }^{3}$ \\ Elena Fiabane ${ }^{2,4}$ \\ 'Miller Institute for Behavioral \\ and Cognitive Therapy, Genoa, \\ Italy; ${ }^{2}$ Department of Physical and \\ Rehabilitation Medicine, ICS Maugeri \\ Spa SB, Institute of Genoa Nervi, \\ Genoa, Italy; ${ }^{3}$ Psychology Unit, \\ ICS Maugeri SPA SB, IRCCS, Tradate, \\ Italy; ${ }^{4}$ Department of Brain and \\ Behavioral Sciences, University of \\ Pavia, Pavia, Italy
}

\begin{abstract}
Purpose: The aims of this pilot study were to examine cognitive factors (brooding and craving) together with positive/negative metacognitive beliefs about alcohol during a residential program for alcohol addiction and to explore relationships with psychological variables at discharge, with the scope of identifying predictive factors of psychological outcome and patients at greatest risk of relapse.
\end{abstract}

Methods: Thirty patients underwent a brief semistructured interview on admission to a 28-day rehabilitation program for alcohol addiction, and completed at admission and discharge the following five self-report questionnaires: 1) brooding (Brooding subscale of Ruminative Response Scale [B-RRS]), 2) craving (Penn Alcohol Craving Scale [PACS]), 3) positive beliefs about alcohol use (Positive Alcohol Metacognitions Scale [PAMS]), 4) negative beliefs about alcohol use (Negative Alcohol Metacognitions Scale [NAMS]), and 5) the psychophysical state of health (Cognitive Behavioral Assessment - Outcome Evaluation [CBA-OE]).

Results: Significant changes were found between admission and discharge in CBA-OE, B-RRS, and PACS. Brooding at admission was a significant predictor of post-treatment psychological variables of "anxiety", "depression", and "psychological distress", whereas craving at admission was a good predictor of "perception of positive change" at discharge.

Conclusion: Our results confirm the importance of brooding in mood regulation and its role in the development and maintenance of problem drinking. In addition, craving was negatively associated with the perception of positive change in the post-treatment outcomes and was a predictor of this psychological variable, which includes features related to the individual's resilience and strength. The changes in metacognitive beliefs regarding alcohol use were not statistically significant, but we found a reduction in positive metacognitions and an increase in negative alcohol-related beliefs; future studies are needed to further explore this issue.

Keywords: alcohol addiction, cognitive factors, metacognition, rehabilitation, craving, brooding

\section{Introduction}

Alcohol is one of the world's top priorities in the area of public health and a major cause of illness and premature death. In Europe, it is the third leading risk factor for disease and mortality after tobacco smoking and hypertension. ${ }^{1}$ Alcohol is a well-known cause of diverse health problems including injuries, mental and behavioral disorders, gastrointestinal conditions, cancer, cardiovascular disease, immunological disorders, lung disease, skeletal and muscular diseases, genital disorders, and fetal harm. ${ }^{2}$ Because addiction involves so many aspects of an individual's life, treatment is not simple.
Correspondence: Marcella Ottonello Department of Physical and Rehabilitation Medicine, ICS Maugeri Spa SB, Institute of Genoa Nervi, Via Missolungi, I4, Genoa 16167, Italy

Tel +39 I0 3079 |25|

Email marcella.ottonello@icsmaugeri.it $\mathrm{BY}$
hC hereby accept the Terms. Non-commercial uses of the work are permitted without any further permission from Dove Med 
Treatment options for alcoholism can be performed in a residential hospital setting, as long-term residential treatment (therapeutic community), or through an outpatient program. ${ }^{3}$ Effective treatment programs typically incorporate different components, each targeting a particular aspect of the illness and its consequences. Addiction treatment must help the individual to reach abstinence, change lifestyle, and achieve productive functioning in the family, on the job, and in the society. ${ }^{4}$ The wide variety of current treatment options includes drugs (eg, disulfiram), cognitive behavioral therapy (CBT), motivational reinforcement techniques, 12-step facilitation therapy, coping skills' management, relapse prevention therapy, and family therapy. ${ }^{5}$ The inpatient rehabilitation 28-day program ${ }^{6}$ used in this study was inspired by the American Minnesota Model, ${ }^{7}$ Croatian psychiatry studies, ${ }^{8}$ and German and Austrian "psychosomatic clinics". 9 It consisted of a series of highly structured interventions involving group psychotherapy, individual therapy (CBT), alcohol-related risk education sessions, and group activities and group meetings with family members to manage the patient's medical and psychological problems.

Previous studies have shown an overall reduction in anxious-depressive symptoms and a psychological improvement among alcoholics during the first weeks after detoxification. ${ }^{6,10,11}$ However, some authors have found that the period after detoxification is at great risk of depressive symptoms and relapses and research is needed to examine factors associated with the maintenance of change and abstinence. ${ }^{10}$

There is a large body of literature exploring the relationship between alcohol consumption and metacognitive processes as a factor that causes dysfunctional thinking processes, which can generate and maintain psychological disorders. ${ }^{12-14}$ Metacognition refers to the psychological structures, beliefs, events, and processes that are involved in the control, modification, and interpretation of thinking. ${ }^{15}$ Specifically, alcohol use has been considered as a strategy for controlling negative emotions and thoughts, which are influenced by metacognitions. ${ }^{13}$ The great majority of theorists have differentiated between the following two basic aspects of metacognition: metacognitive regulation and metacognitive knowledge (or metacognitive beliefs). In the metacognitive conceptualization of psychological dysfunction, both constructs interact in maintaining maladaptive behaviors. $^{15,16}$

Research has identified the existence of positive metacognitive beliefs about alcohol use, ${ }^{17}$ conceptualized as a specific form of outcome expectancy relating to the use of alcohol (eg, "drinking makes me think more clearly" and "drinking reduces my anxious feelings"), and negative metacognitive beliefs such as perception of lack of executive control over alcohol use and of the cognitive costs of drinking (eg, "drinking controls my life" and "drinking will damage my mind"). Among the numerous mechanisms that may underlie craving, a cognitive-affective model has suggested an interaction between addiction and affective symptoms. ${ }^{18}$

Specifically, negative affectivity turned out to be an important antecedent of craving in clinical studies. ${ }^{19,20}$ Since craving is a strong predictor of relapse after detoxification, ${ }^{21}$ the relationships among psychological changes, craving, and negative moods during the treatment of alcohol dependence need to be investigated in order to identify patients at high risk of relapse. Another cognitive factor, brooding (a dimension of rumination), should also be explored as several studies have suggested that rumination is a vulnerability factor for depression and a core cognitive process in the pathology of mood disorders. Brooding is the tendency to passively compare individual current situations with unachieved goals and may represent a maladaptive aspect of rumination with respect to the longitudinal prediction of depression. ${ }^{22}$ According to the self-regulatory function model, anxiety and depression are also associated in dysfunctional metacognitive dimensions. ${ }^{15,23}$

In the light of these considerations and to provide information on feasibility of a full-scale study, we aimed to evaluate changes over time in metacognitive (positive and negative) beliefs about alcohol use and cognitive factors (craving and brooding) in a sample of alcohol-dependent patients undergoing a residential rehabilitation program, to analyze whether these changes are influenced by individual sociodemographic and clinical characteristics, and to explore baseline metacognitive and cognitive factors as possible predictors of the psychological outcomes at discharge (anxiety, depression, psychological distress, and perception of positive change) in order to identify patients at greatest risk of relapse in this pilot study.

\section{Patients and methods}

\section{Patients}

The preliminary study was carried out in 30 patients who underwent a 28-day rehabilitation program for alcohol addiction at the Department of Physical and Rehabilitation Medicine, ICS Maugeri Spa SB, Institute of Genoa Nervi, Genoa, Italy. Inclusion criteria were as follows: age $>18$ years, current diagnosis of alcohol dependence according to the Diagnostic and Statistical Manual of Mental Disorders, fourth edition, text revision (DSM-IV-TR), ${ }^{24}$ absence of dementia (evaluated with Mini-Mental State 
Examination $\geq 24$ ), understanding of the Italian language (spoken or written if patients of other nationalities), and participation in $\geq 80 \%$ of the proposed rehabilitation sessions. An exclusion criterion was the presence in the anamnestic evaluation of antisocial personality disorder because that antisocial personality disorder was not suitable for group therapy and residential treatment. Of the 47 patients initially screened, 14 (29.7\%) patients were excluded because they did not meet the inclusion criteria, and a further three $(6.3 \%)$ patients were later excluded from analysis because of missing responses on the questionnaires (Figure 1). The final sample consisted of 30 patients who were informed about the aims of the study and gave their written informed consent. All data collected in this study were derived from tools and treatments used in routine clinical practice at our institute. This is a preliminary study of a research project approved by the Regional Ethics Committee of the IRCCS AOU San Martino-IST (PR 430Reg2015).

\section{Procedure}

Following admission to the rehabilitation unit, patients underwent a brief initial interview with an addiction medicine physician or psychiatrist with wide experience in managing addiction, which served to guide medical management during detoxification and discharge planning. Sociodemographic and clinical data about the patient were also gathered through the interview, for example, age, level of education, work status, duration of alcohol dependence, and possible presence of other drug dependences or psychiatric comorbidities. At the end of their first week of residential treatment, after complete detoxification (pretreatment, t0), patients were administered a battery of five self-report questionnaires; this battery was then re-administered 3 days before their hospital discharge (posttreatment, $\mathrm{t} 1$ ). The residential program (suited for patients in severe clinical condition but with a low level of social

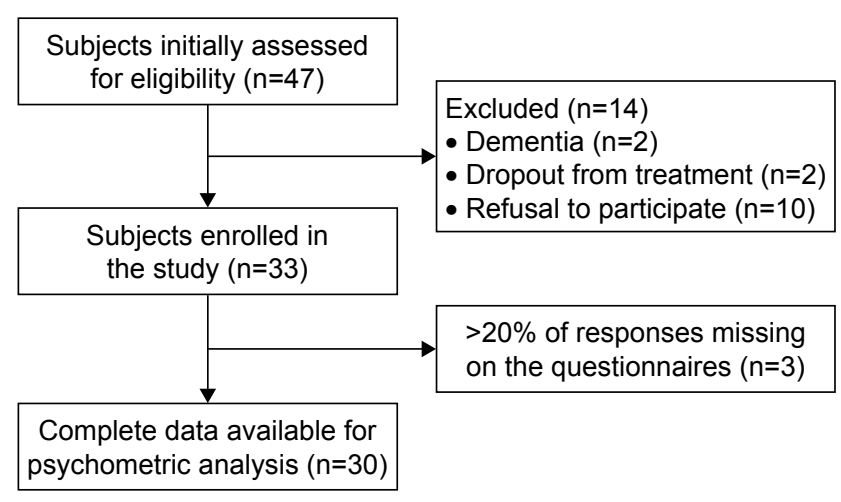

Figure I Flowchart showing the patient enrolment process. problems) lasted 28 days and consisted of high-intensity medical and psychotherapeutic interventions, comprising

- Multiprofessional team interventions (including medical, nursing, psychological, and educational) on the needs of the individual;

- Evaluation and treatment of acute withdrawal symptoms, which may range from mild (eg, tremor and insomnia) to severe (eg, autonomic hyperactivity, seizures, and delirium), through an evaluation of withdrawal severity and symptoms' therapy with drugs;

- CBT training focused on restructuring beliefs, attributions, and expectations that are related to excessive alcohol use, acquiring coping skills to manage high-risk drinking situations and comorbid psychological problems;

- Group therapy to support and maintain the motivation to change, to educate on alcohol-related risks, and to promote the activation of interpersonal skills;

- Group activities with various modes of expression, such as movie discussions, gymnastics, and theme-based groups (ie, managing emotions and self-esteem promotion);

- Personal involvement in the care of the patients, encouraging patients to take care of themselves and their environment (ie, keeping their room tidy and making their bed); and

- Group meetings with family members (if available) to provide support, counseling, and involve them in the rehabilitation program.

During residential rehabilitation, patients were required to attend 1-hour group therapy sessions twice daily regarding the maintenance of abstinence, the education of alcohol-related risks, motivation to change, CBT training, and relapse prevention. Group activities (eg, movie discussions, gymnastics, relaxation techniques, and art therapy) took place in the afternoon.

\section{Measures}

The evaluation tools consisted of a brief initial semistructured interview and a battery of five self-report questionnaires investigating the following areas: 1) brooding, 2) craving, 3) positive beliefs about alcohol use, 4) negative beliefs about alcohol use, and 5) the psychophysical state of health. The battery of self-report questionnaires was administered twice to the patient: at admission to the rehabilitation program and again 3 days before discharge.

The following assessment scales were used.

\section{Brooding subscale of Ruminative Response Scale (B-RRS)}

The Ruminative Response Scale (RRS) is a self-report instrument that measures individual differences in the tendency to 
ruminate in response to negative affect. ${ }^{25,26}$ For this study, we used the B-RRS made up of five items addressing the brooding dimension of rumination (eg, "Think 'What did I do to deserve this?"'). ${ }^{26}$ The questionnaire instructions ask people to focus their attention on the thoughts they have when they are "sad, melancholic, or depressed" and rate on a 4-point scale (from 1 "almost never" to 4 "almost always") how often they experience what is indicated by each item. This subscale has been used in previous studies ${ }^{27}$ and showed good psychometric proprieties. ${ }^{26}$ The internal consistency (Cronbach's $\alpha$ ) of the B-RRS was 0.77, and the test-retest reliability measured during 2 years was $0.62 .{ }^{26}$

\section{Penn Alcohol Craving Scale (PACS)}

The PACS is a 5-item scale developed to assess various aspects of craving for alcohol over the past week. ${ }^{28}$ The first three questions concern the frequency, intensity, and duration of drink-related thoughts. The fourth question assesses the ability to refrain from drinking if alcoholic beverages are available. The last question invites the subject to make an estimate of the alcohol craving over the previous week. PACS has proven to be a useful and reliable tool for measuring craving and is a predictor of the risk of relapse. Items are evaluated on a frequency scale from 0 to 6. The PACS has been shown to possess good psychometric properties in terms of reliability (Cronbach's $\alpha=0.92$ ) and concurrent, predictive, and discriminant validities. ${ }^{28}$

\section{Positive Alcohol Metacognitions Scale (PAMS)}

The PAMS is a 12-item scale assessing positive metacognitions about alcohol use. ${ }^{17}$ It consists of the following two factors: 1) positive alcohol beliefs about emotional self-regulation and 2) positive alcohol beliefs about cognitive self-regulation. Participants are asked to rate how much they agree with each statement, referring to when they begin drinking, on a Likert scale from 1 (do not agree) to 4 (agree very much). Higher scores represent higher levels of positive metacognitive belief about alcohol use. Examples of positive metacognitive beliefs about alcohol use may include "drinking makes me think more clearly" (problem solving), "drinking helps me to control my thoughts" (thought control), "drinking helps me focus my mind" (attention regulation), "drinking reduces my self-consciousness" (self-image control), and "drinking reduces my anxious feelings" (emotion regulation). This scale is dimensional and has shown good psychometric qualities of internal and external reliabilities in both community and clinical populations. ${ }^{17}$

\section{Negative Alcohol Metacognitions Scale (NAMS)}

The NAMS is a 6-item scale assessing negative metacognitions about alcohol use. It consists of the following two factors: 1) negative alcohol beliefs about uncontrollability and 2) negative alcohol beliefs about cognitive harm. Participants are asked to rate how much they agree with each statement, referring to when they have stopped a session of drinking, on a Likert scale from 1 (do not agree) to 4 (agree very much). Higher scores represent higher levels of negative metacognitive belief about alcohol use. Negative beliefs concern the significance, uncontrollability, and danger of thoughts. Examples include "I need to control my thoughts otherwise they will control me" or "my thoughts of harm will make me lose my mind". NAMS is dimensional and possesses good internal and external reliabilities in both community and clinical populations. ${ }^{17}$

To assess the state of psychophysical health, we used the following questionnaire.

\section{Cognitive Behavioral Assessment - Outcome Evaluation (CBA-OE)}

The CBA-OE was the questionnaire used to evaluate psychological treatment effects during the residential program. ${ }^{29}$ The questionnaire has 80 items and uses a 5-point scale, ranging from $1=$ nothing to $5=\mathrm{a}$ lot. The CBA-OE consists of the following five scales: anxiety (14 items, eg, "I have been upset about trivial things"), well-being (15 items, eg, "I have done things that interested and involved me"), perception of positive change (11 items, eg, "I have tried to deal with difficulties rather than avoid them"), depression (19 items, eg, "I have been tormented by feelings of guilt"), and psychological distress (21 items, eg, "I have felt debased or mocked"). In answering each item, respondents refer to how they felt over the previous 2 weeks. The questionnaire has demonstrated excellent psychometric properties: it has a strong factor structure, good reliability (Cronbach's $\alpha$ between 0.80 and 0.91 for the clinical sample), criterion-related and convergent validities, and the power to discriminate between nonclinical and "suffering-distressed" subjects. ${ }^{29,30}$

\section{Statistical analyses}

We used descriptive statistics - number (percentage) or mean \pm standard deviation (SD) - to present the basic characteristics of the study participants. The scores on the subscales of CBA-OE, PAMS, NAMS, B-RRS, and PACS at $\mathrm{t} 0$ and $\mathrm{t} 1$ are reported as mean $\pm \mathrm{SD}$. The assumptions of multivariate normality and linearity were evaluated through SPSS.

Cognitive scales were analyzed in terms of changes from admission to discharge on single items. We considered items as interesting that had scores higher than the average response values for each item. We investigated significant differences in psychological and metacognitive variables between admission 
and discharge through Student's $t$-test (nondirectional, $\alpha=0.05$ ). The size effect was also calculated using Cohen's $d$ statistic, ${ }^{31}$ and Sawilowsky (2009) ${ }^{32}$ outlined the criteria for assessing small, medium, and large effect sizes in different metrics, such as $d$-effect small $\geq 0.20$, medium $\geq 0.50$, large $\geq 0.80$, and very large $\geq 1.20$. Possible correlations between psychological and metacognitive variables were calculated with Pearson's correlation coefficient. We also analyzed differences in metacognitive beliefs according to patients' sex, level of education, work status (employed vs unemployed), substance dependence (alcohol vs polysubstance), type of intervention (pharmaco- and psychotherapy vs psychotherapy only), and previous admissions for alcohol dependence (yes vs no) through one-way analysis of variance (ANOVA). Pearson's correlation analysis was performed to examine bivariate correlations among cognitive beliefs (eg, PAMS, PACS, and B-RRS) and post-treatment variables of CBA-OE. Variables showing a significant correlation with post-treatment psychological outcomes $(P \leq 0.05)$ were included simultaneously in a multivariate regression model to identify baseline predictors of post-treatment psychological outcomes. A $P$-value of 0.05 was considered as statistically significant. All statistical analyses were conducted using SPSS, Windows Version 13.0 (SPSS Inc., Chicago, IL, USA).

\section{Results}

Patients were predominantly male (70\%) and had a mean age of $50.03 \pm 10.52$ years. The majority was unemployed $(60 \%)$ and had a middle-high education level (63.3\%). Most patients had a history of $>10$ years of alcohol dependence (70\%), and most (80\%) were not polysubstance dependent. Patients' sociodemographic and clinical characteristics are shown in Table 1.

Results of the CBA-OE scales and metacognitive/ cognitive beliefs scales at admission and discharge are reported in Table 2. We found significant differences between admission and discharge in all CBA-OE variables as well as in the B-RRS and PACS. In addition, exploring changes from admission to discharge on single items of the metacognitive and cognitive scales (Figures 2 and 3), we found that

1) At admission, all items of PAMS had higher scores than the average score for that item (mean value 2 ) excepting items 1 (drinking makes me more affectionate), 3 (drinking makes me think more clearly), 5 (drinking helps me to control my thoughts), and 11 (drinking helps me focus my mind);

2) At discharge, scores were lower on all items with the exception of items 5 (drinking helps me to control my thoughts) and 9 (drinking reduces my self-consciousness),
Table I Sociodemographic and clinical characteristics of the total sample $(n=30)$

\begin{tabular}{|c|c|}
\hline Variable & n (\%) \\
\hline \multicolumn{2}{|l|}{ Sex } \\
\hline Male & $21(70)$ \\
\hline Female & $9(30)$ \\
\hline \multicolumn{2}{|l|}{ Education } \\
\hline Low & II (36.7) \\
\hline Middle-high & $19(63.3)$ \\
\hline \multicolumn{2}{|l|}{ Work status } \\
\hline Employed & $18(60)$ \\
\hline Not employed & $12(40)$ \\
\hline \multicolumn{2}{|l|}{ Comorbidity } \\
\hline Yes & $17(56.7)$ \\
\hline No & $13(43.3)$ \\
\hline \multicolumn{2}{|l|}{ Intervention } \\
\hline Pharmaco- and psychotherapy & $20(64.5)$ \\
\hline Psychotherapy only & $10(32.3)$ \\
\hline \multicolumn{2}{|l|}{ Alcohol dependence } \\
\hline$\leq 10$ years & $9(30)$ \\
\hline$>10$ years & $21(70)$ \\
\hline \multicolumn{2}{|l|}{ Polysubstance dependence } \\
\hline Pure alcoholics & $24(80)$ \\
\hline Polyabusers & $6(20)$ \\
\hline \multicolumn{2}{|l|}{ Previous admissions } \\
\hline Yes & $17(56.7)$ \\
\hline No & $13(43.3)$ \\
\hline \multicolumn{2}{|l|}{ Age (years) } \\
\hline Mean \pm SD & $50.03 \pm 10.52$ \\
\hline
\end{tabular}

Abbreviation: SD, standard deviation.

which increased slightly. The items that changed most from admission to discharge (with an increase of $>0.5$ ) were items 4 (drinking makes me feel more relaxed), 6 (drinking makes my negative thoughts more bearable), and 7 (drinking reduces my anxious feelings);

3) Similarly, all items of NAMS, at admission, had scores higher than the average score (value 2 ). The scores on three items (2, 3, and 5) increased after the rehabilitation program, in particular item 2 (if I cannot control my drinking, I will cease to function). At discharge, only item 1 (I have no control over my drinking) and item 6 (drinking controls my life) decreased slightly;

4) Concerning B-RRS, scores on all items decreased after treatment, in particular items 2 (why do I always react this way?) and 3 (think about a recent situation, wishing it had gone better);

5) For PACS, all item scores decreased at discharge. The main changes were for items 1 (during the past week how often have you thought about drinking?) and 2 (how strong was your craving during the past week?).

Significant differences in findings of the four questionnaires (PAMS, NAMS, B-RRS, and PACS) between patients based on their sociodemographic and clinical characteristics 
Table 2 Changes in mean scores of psychological factors and cognitive-metacognitive scales at admission to discharge from the rehabilitation program (total patients $=30$ )

\begin{tabular}{|c|c|c|c|c|c|}
\hline Variable & Admission, mean (SD) & Discharge, mean (SD) & $t$ & $P$-value & Cohen's d \\
\hline \multicolumn{6}{|l|}{ CBA-OE subscales } \\
\hline Anxiety & $24.77(10.78)$ & $12.70(7.25)$ & 7.002 & $<0.001$ & -1.11 \\
\hline Well-being & $22.33(10.84)$ & $34.53(7.87)$ & -6.053 & $<0.00$ I & 1.12 \\
\hline Perception of positive change & $20.73(6.66)$ & $27.53(5.37)$ & -6.546 & $<0.00$ I & 1.02 \\
\hline Depression & $32.70(13.46)$ & $14.87(8.10)$ & 8.177 & $<0.001$ & -1.32 \\
\hline Psychological distress & $30.40(15.5 I)$ & $12.03(11.00)$ & 7.588 & $<0.00 \mathrm{I}$ & -1.18 \\
\hline \multicolumn{6}{|l|}{ Cognitive and metacognitive scales } \\
\hline PAMS & $26.66(8.18)$ & $23.70(7.33)$ & 2.022 & 0.052 & -0.36 \\
\hline NAMS & I6.23 (4.08) & $15.70(4.22)$ & 0.77 & 0.448 & -0.12 \\
\hline B-RRS & $|2.6|(3.33)$ & $10.96(3.28)$ & 3.601 & $<0.00$ I & -0.50 \\
\hline PACS & $17.86(6.47)$ & $11.06(7.54)$ & 5.302 & $<0.001$ & -1.05 \\
\hline
\end{tabular}

Abbreviations: B-RRS, Brooding subscale of Ruminative Response Scale; CBA-OE, Cognitive Behavioral Assessment - Outcome Evaluation; NAMS, Negative Alcohol Metacognitions Scale; PACS, Penn Alcohol Craving Scale; PAMS, Positive Alcohol Metacognitions Scale; SD, standard deviation.

are reported in Table 3 . With regard to sex and work status, we found significant differences at admission on the PAMS $(P<0.05)$. We also found differences on NAMS at admission and discharge depending on the type of therapy (pharmaco- and psychotherapy vs psychotherapy only) $(P<0.05)$. Concerning previous admissions, there were significant differences on PACS at admission $(P<0.05)$.
Results of the correlation analysis (Table 4) showed significant correlations between B-RRS and "anxiety", "depression", and "psychological distress" subscales of CBA-OE. A significant correlation was found between PACS and the CBA-OE subscale "perception of positive change". At a multivariate linear regression analysis of predictors of post-treatment outcomes, the B-RRS at admission resulted

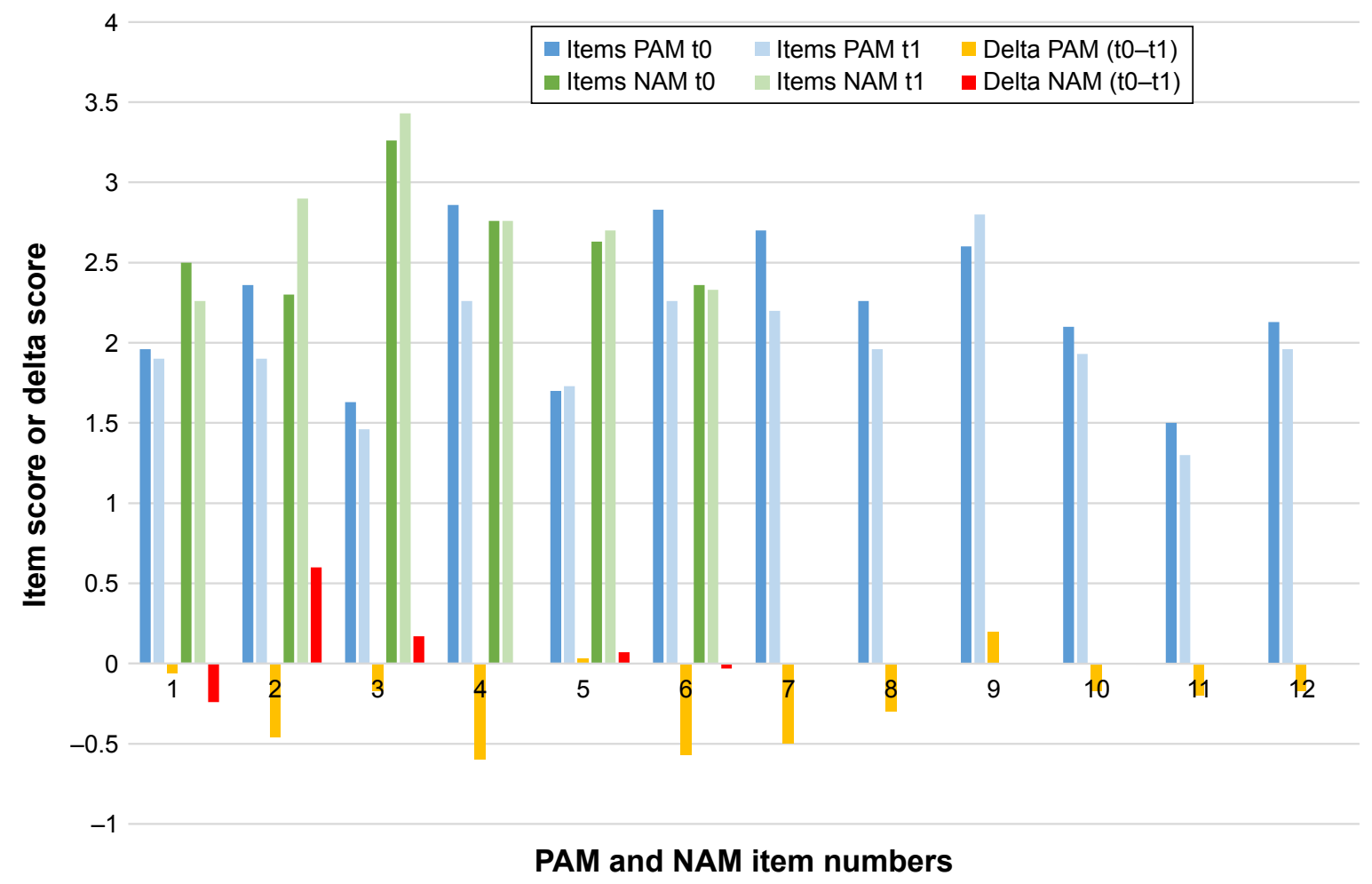

Figure 2 PAM and NAM at admission ( $\mathrm{t} 0$ ) and discharge $(\mathrm{tl})$, with changes between the two evaluations.

Notes: PAM items: I, Drinking makes me more affectionate; 2, Drinking makes me more confident; 3, Drinking makes me think more clearly; 4, Drinking makes me feel more relaxed; 5, Drinking helps me to control my thoughts; 6 , Drinking makes my negative thoughts more bearable; 7 , Drinking reduces my anxious feelings; 8 , Drinking makes me more sociable; 9, Drinking reduces my self-consciousness; 10, Drinking makes me feel happy; II, Drinking helps me focus my mind; I2, Drinking helps me fit in socially. NAM items: I, I have no control over my drinking; 2, If I cannot control my drinking I will cease to function; 3, Drinking will damage my mind; 4, My drinking persists no matter how I try to control it; 5, Drinking will make me lose control; 6, Drinking controls my life.

Abbreviations: NAM, negative alcohol metacognitions; PAM, positive alcohol metacognitions. 


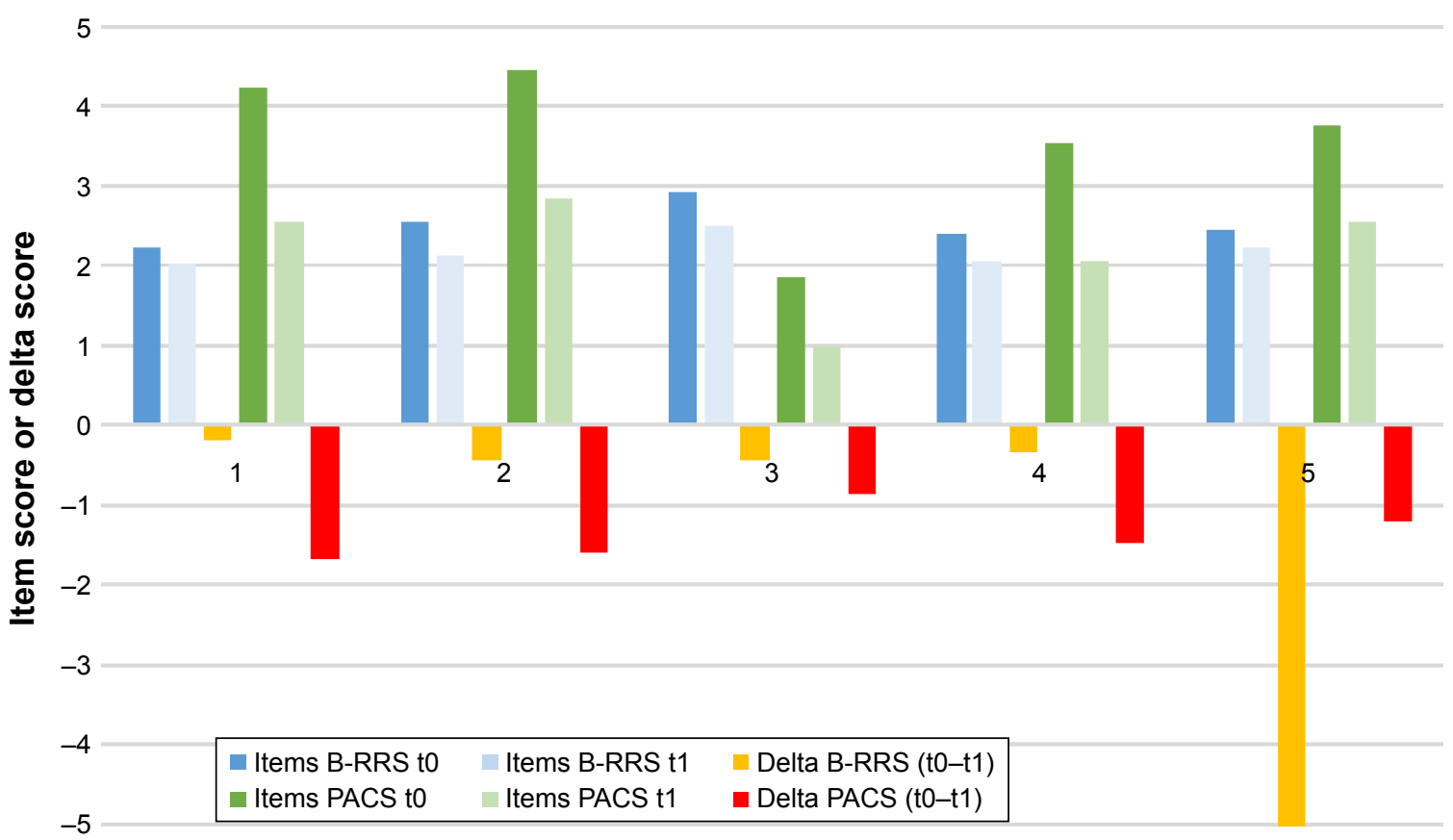

PAM and NAM item numbers

Figure 3 B-RRS and PACS at admission ( $\mathrm{t} 0$ ) and discharge $(\mathrm{tl})$, with changes between the two evaluations.

Notes: B-RRS items: I, Think "What am I doing to deserve this?"; 2, Think "Why do I always react this way?"; 3, Think about a recent situation, wishing it had gone better; 4, Think "Why do I have problems other people don't have?"; 5, Think "Why can't I handle things better?". PACS items: I, During the past week how often have you though about drinking or about how good a drink would make you feel?; 2, At its most severe point, how strong was your craving during the past week?; 3, During the past week how much time have you spent thinking about drinking or about how good a drink would make you feel?; 4, During the past week how difficult would it have been to resist taking a drink if you had known a bottle were in your house?; 5, Keeping in mind your responses to the previous questions, please rate your overall average alcohol craving for the past week.

Abbreviations: B-RRS, Brooding subscale of Ruminative Response Scale; PACS, Penn Alcohol Craving Scale.

to be a significant predictor of depression and psychological distress, while the PACS at admission was a significant predictor of perception of positive change (Table 5).

\section{Discussion}

The first aim of this pilot study was to provide information about whether a full-scale study on cognitive and metacognitive factors among alcohol-dependent patients during residential rehabilitation would be feasible and worthwhile. In particular, we analyzed how these factors changed over time, during residential rehabilitation treatment for alcohol addiction and if we could predict an individual's psychological outcome, eg, the perception of a positive change (need to get support from others and ability to cope with difficulties) or psychological distress or serious mood symptoms of disorder, based on them. Previous research has shown that negative mood increases the urge to drink alcohol, ${ }^{33}$ and the motivation to drink as a mean of reducing the negative affect is incorporated into psychological theories of substance abuse. ${ }^{34}$ Similarly, research has demonstrated a positive association between alcohol craving and alcohol use and has identified craving as a central component of alcohol use disorder. ${ }^{35}$ Further research has identified the existence of specific positive and negative metacognitive beliefs that play a central role in motivating individuals to engage in alcohol use as a mean of cognitive emotional regulation. ${ }^{36}$

Concerning psychological functioning, our findings showed an overall significant improvement in all scales of the CBA-OE questionnaire, highlighting the psychological improvements of patients during the detoxification process and rehabilitation program. Residential rehabilitation programs for alcohol disorders are in fact designed to detoxify the individual, to enhance their motivation to change, coping strategies, and interpersonal skills, and to modify dysfunctional beliefs. ${ }^{37}$ The scores after treatment on the scales of anxiety, depression, and psychological distress were diminished. These findings are in line with the current literature, which shows a decrease in anxiety disorders and negative affect, including depressive states, following alcohol

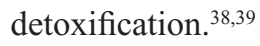

Metacognitive beliefs about alcohol use did not show significant changes during rehabilitation. Nevertheless, positive metacognitive beliefs about alcohol use had lower scores after treatment except for the following two items: drinking helps me to control my thoughts (thought control) and drinking reduces my self-consciousness (self-image regulation). 
The positive beliefs that showed most change concerned emotion regulation (drinking reduces my anxious feelings) and mood regulation (drinking makes my negative thoughts more bearable and drinking makes me feel more relaxed). Regarding the negative metacognitive beliefs, we found an improvement in beliefs about cognitive harm associated with alcohol (if I cannot control my drinking, I will cease to function, drinking will damage my mind, and drinking will make me lose control), a probable consequence of education on alcohol-related risks.

Women generally had lower scores than men on positive beliefs related to alcohol use except for item 6 (drinking makes my negative thoughts more bearable). Women's general lower positive beliefs about cognitive self-regulation of alcohol and their belief that alcohol use is a helpful strategy when dealing with negative thoughts confirm the known greater presence of significant depressive symptoms in alcoholic women. ${ }^{40}$ Another sociodemographic variable evaluated was the influence of work status on cognitive/metacognitive factors during the program. Unemployed patients showed a higher level of positive beliefs about alcohol use, in particular concerning the ability of alcohol to modulate anxiety and negative effect. Research shows a clear link between precarious employment or unemployment and depression. ${ }^{41}$

The influence of the type of therapy performed (pharmaco- and psychotherapy vs psychotherapy only) on negative alcohol metacognition scores at admission and discharge confirmed that patients who received only psychotherapy (no pharmacotherapy) responded better to cognitive behavioral treatment and the educational intervention. Those who received drug therapy for withdrawal syndrome, craving, or mental health symptoms had more severe symptoms at admission, which would explain the need for this choice of intervention. A significant difference on the PACS at admission was found in relation to previous admissions. Frequency and intensity of craving were greater in those who had previous hospitalizations for alcohol detoxification. This finding is in line with a large body of research showing the association between alcohol craving and alcohol intake, the severity of alcohol dependence, and recurrent detoxifications. ${ }^{42-44}$

The significant change in B-RRS in terms of the lower self-reported rumination may be related to an improvement in the mood of patients during the program. In fact, rumination appears to contribute to a sense of hopelessness about the future and negative evaluations of the self and it represents one of the core cognitive processes in the pathology of depressive disorders. ${ }^{45}$ In particular, "brooding" as a passive comparison of one's current situation with some unachieved standard seems to represent a maladaptive aspect 
Table 4 Correlations among post-treatment CBA-OE subscales and pretreatment cognitive and metacognitive factors

\begin{tabular}{|c|c|c|c|c|c|c|c|c|c|}
\hline Variable & $\mathbf{I}$ & 2 & 3 & 4 & 5 & 6 & 7 & 8 & 9 \\
\hline I. Positive alcohol metacognitions & I & & & & & & & & \\
\hline 2. Negative alcohol metacognitions & $0.4 I^{*}$ & I & & & & & & & \\
\hline 3. Craving & 0.25 & 0.11 & 1 & & & & & & \\
\hline 4. Brooding & -0.02 & 0.09 & 0.07 & I & & & & & \\
\hline 5. Anxiety & 0.04 & -0.05 & 0.29 & $0.58 * *$ & 1 & & & & \\
\hline 6. Well-being & -0.09 & 0.11 & -0.22 & -0.23 & -0.33 & I & & & \\
\hline 7. Perception of positive change & -0.07 & 0.23 & $-0.42^{*}$ & -0.24 & $-0.44^{*}$ & $0.64 * *$ & I & & \\
\hline 8. Depression & -0.07 & -0.08 & 0.24 & $0.58 * *$ & $0.78^{* *}$ & -0.32 & $-0.56 * *$ & I & \\
\hline 9. Psychological distress & -0.03 & 0.14 & 0.11 & $0.55 * *$ & $0.75^{* *}$ & -0.04 & -0.25 & $0.72 * *$ & I \\
\hline
\end{tabular}

Notes: Data are presented as Pearson's correlation coefficient $(r)$. $* P$-value $<0.05$. ${ }^{* * P}$-value $<0.00$ I.

Abbreviation: CBA-OE, Cognitive Behavioral Assessment - Outcome Evaluation.

of rumination, ${ }^{46}$ being associated with depression both concurrently and in longitudinal analyses. ${ }^{26}$ In our study, the items that decreased most during the program were those on self-critical thoughts: items 2 (why do I always react this way) and 3 (think about a recent situation, wishing it had gone better). As expected, we found strong correlations between the brooding dimension and anxiety and depression and psychological distress. Rumination is a well-established risk factor for the onset and maintenance of depression ${ }^{47}$ and anxiety symptomatology..$^{48}$ There is growing evidence of the role of brooding as a more maladaptive aspect of rumination, ${ }^{26,46,49}$ and our findings that the brooding score at admission is a predictor of anxiety, depression, and psychological distress in patients at discharge seem to confirm this maladaptive component of rumination. Patients who had a more brooding style of responding to their distress experienced more symptoms of anxiety, depression, and psychological distress. Such patients may benefit from CBT to correct negative thinking styles and reduce ruminative response styles. As this thinking style is a component of cognitive vulnerability for the onset of clinical depression and psychological distress, brooding may also permit to identify those subjects needing further treatment and social support after discharge.

Another factor analyzed was craving, which is thought to play an important role in alcohol use disorder and is correlated with abstinence violation. ${ }^{50}$ All items showed a significant decrease in score at discharge, particularly with respect to the frequency and intensity of craving. Our findings support studies where craving was found to decrease during an abstinence period ${ }^{44,51}$ and are consistent with the motivational model of craving suggesting that craving drives the decision to drink. ${ }^{52}$ The decision to make a change (whether to approach or avoid drinking) tends to resolve the ambivalence and, therefore, reduces the substance-related craving. We found that patients with a low baseline experience of craving achieved a more significant perception of positive change at discharge. Craving was a predictor of this psychological dimension, which comprises features related to the individual's resilience and strength.

Our study has some limitations. First, as a pilot study, it has a small number of participants, limiting the power of the evidence and the generalization of results in multiple comparisons of variables. It means that we can be less confident that there are no significant differences between potential variables, eg, in the case of positive and negative alcohol metacognitions. Despite this, the results showed that a fullscale study is feasible and that the crucial components of the study have been identified. In particular, craving, brooding, and metacognitive beliefs about alcohol use represent separate variables that may account for heterogeneity in the alcohol-related emotive process and cognitive style after treatment. These factors may influence therapy and treatment outcomes. Second, our findings were in the context of a 28-day residential rehabilitation program for alcohol

Table 5 Multivariate regression model for predictors of post-treatment psychological variables

\begin{tabular}{|c|c|c|c|c|c|c|c|c|}
\hline \multirow[t]{2}{*}{ Variable } & \multicolumn{2}{|c|}{ Anxiety } & \multicolumn{2}{|c|}{ Depression } & \multicolumn{2}{|c|}{$\begin{array}{l}\text { Perception of } \\
\text { positive change }\end{array}$} & \multicolumn{2}{|c|}{$\begin{array}{l}\text { Psychological } \\
\text { distress }\end{array}$} \\
\hline & B & $P$ & B & $P$ & B & $\mathbf{P}$ & B & $P$ \\
\hline Positive alcohol metacognitions & 0.05 & 0.70 & -0.06 & 0.69 & -0.08 & 0.51 & -0.13 & 0.60 \\
\hline Negative alcohol metacognitions & -0.30 & 0.31 & -0.28 & 0.41 & 0.47 & 0.06 & 0.32 & 0.51 \\
\hline Craving & 0.28 & 0.11 & 0.29 & 0.15 & -0.34 & 0.02 & 0.15 & 0.60 \\
\hline Brooding & 1.27 & 0.001 & $\mathrm{I} .40$ & 0.001 & -0.40 & 0.14 & 1.76 & 0.003 \\
\hline
\end{tabular}

Notes: $B$, unstandardized coefficient; $P$, Pearson correlation coefficient. 
dependence in which patients were detoxified and committed to joining the treatment, and hence, our results cannot be generalized to alcohol-dependent individuals in other contexts. Third, some important factors associated with alcohol dependence (eg, biological markers, neuropsychological evaluation, and additional behavior assessment), which might have influenced the results, were not explored in this study. Finally, the lack of a control group does not allow us to confirm that psychological improvements are associated with the treatment; however, our aim was to explore psychological changes and not to test the effectiveness of a specific treatment. Nevertheless, the results of this pilot study add weight to the notion that metacognitive and cognitive variables are relevant to understanding alcohol use disorders and should be furthered investigated in a larger study sample (to avoid potentially biased results).

\section{Conclusion}

Our preliminary study showed that in-hospital rehabilitation for alcohol dependence is associated with an overall improvement in all psychological variables measured with CBA-OE and with craving and brooding. In addition, we found that baseline craving is negatively associated with the perception of positive change in the post-treatment outcomes and is a predictor of this psychological variable, which includes features related to the individual's resilience and strength. Our results also showed that brooding is a significant predictor of anxiety, depression, and psychological distress, supporting the importance of this particular dimension of rumination in mood regulation, given the strong association between negative emotions, craving, and alcohol use. These preliminary findings suggest the need for an early assessment of craving and brooding in alcohol dependence and a careful monitoring of these cognitive factors during rehabilitation programs to better orient treatment in order to achieve positive outcomes.

\section{Disclosure}

The authors report no conflicts of interest in this work.

\section{References}

1. World Health Organization. Global Health Adds Life to Years. Global Brief for World Health Day: 2012. Geneva: WHO; 2012.

2. Anderson P, Baumberg B. Alcohol in Europe: A Public Health Perspective. A Report of the European Commission. Vol. 13. London, UK: Institute of Alcohol Studies (IAS); 2006:483-488.

3. Richard K, Fuller MD, Hiller-Sturmhöfel S. Alcoholism treatment in the United States. Alcohol Res Health. 1999;23(2):69-77.

4. National Institute on Alcohol Abuse and Alcoholism (NIAAA). Helping Patients Who Drink Too Much: A Clinician's Guide. 2005 ed. NIH Publication; 1999. NIH Publication No. 12-4180 Printed 1999; Reprinted July 2000, February 2008; Revised April 2009, December 2012.
5. Kadden R. Cognitive Behavioral Coping Skills Therapy Manual: A Clinical Research Guide for Therapists Treating Individuals with Alcohol Abuse and Dependence. Collingdale, PA: Diane Publishing; 1995.

6. Giorgi I, Ottonello M, Vittadini G, Bertolotti G. Psychological changes in alcohol-dependent patients during a residential rehabilitation program. Neuropsychiatr Dis Treat. 2015;11:2989-2996.

7. Anderson DJ, McGovern JP, DuPont RL. The origins of the Minnesota model of addiction treatment - a first person account. J Addict Dis. 1999; 18(1):107-114.

8. Hodolin V, Corlito G. Psichiatria, Alcologia, Riabilitazione. Trento, Italy: Centro Studi Erickson; 1996.

9. Hinnental I, Cibin M. Il Trattamento Residenziale Breve delle Dipendenze da Alcol e Cocaina: Il Modello Soranzo. Turin: SEED; 2011.

10. Driessen M, Meier S, Hill A, Wetterling T, Lange W, Junghanns K. The course of anxiety, depression and drinking behaviours after completed detoxifcation in alcoholics with and without comorbid anxiety and depressive disorders. Alcohol Alcohol. 2001;36(3):249-255.

11. Lookatch SJ, Elledge LC, Anderson S, Shorey RC, Stuart GL, Moore TM. Cognitive and psychological changes during 28-day residential substance use treatment. Addict Res Theory. 2017;25(4):334-341.

12. Spada MM, Zandvoort M, Wells A. Metacognitions in problem drinkers. Cognit Ther Res. 2007;31(5):709-716.

13. Spada MM, Wells A. Metacognitions, emotion and alcohol use. Clin Psychol Psychother. 2005;12(2):150-155.

14. Ipek OU, Yavuz KF, Ulusoy S, Sahin O, Kurt E. Metacognitive and meta-emotional styles in patients with alcohol and the other substance dependence. Int J High Risk Behav Addict. 2015;4(3):e24553.

15. Wells A. Emotional Disorders and Metacognition: Innovative Cognitive Therapy. Chichester, UK: Wiley; 2000.

16. Wells A, Matthews G. Attention and Emotion. A Clinical Perspective. Hove, UK: Erlbaum; 1994.

17. Spada MM, Wells A. Metacognitive beliefs about alcohol use: development and validation of two self-report scales. Addict Behav. 2008;33(4):515-527.

18. Backer TB, Piper ME, McCarthy DE, Majeskie MR, Fiore MC. Addiction motivation reformulated: an affective processing model of negative reinforcement. Psychol Rev. 2004;111(1):33-51.

19. Pombo S, Figueira ML, Walter H, Lesch O. Motivational factors and negative affectivity as predictors of alcohol craving. Psychiatry Res. 2016;243:53-60.

20. Witkiewitz K, Lustyk MKB, Bowen S. Retraining the addicted brain: a review of hypothesized neurobiological mechanisms of mindfulnessbased relapse prevention. Psychol Addict Behav. 2013;27(2):351.

21. Browne KC, Wray TB, Stappenbeck CA, Krenek M, Simpson TL. Alcohol consumption, craving, and craving control efforts assessed daily in the context of readiness to change among individuals with alcohol dependence and PTSD. J Subst Abuse Treat. 2016;61:34-41.

22. Hasegawa A, Koda M, Hattor Y, Kondo T, Kawaguchi J. Longitudinal predictions of the brooding and reflection subscales of the Japanese Ruminative Responses Scale for depression. Psychol Rep. 2013;113(2): 566-585.

23. Papageorgiou C, Wells A. An empirical test of a clinical metacognitive model of rumination and depression. Cognit Ther Res. 2003; 27(3):261-273.

24. American Psychiatric Association. Diagnostic and Statistical Manual of Mental Disorders. 4th ed. Washington, DC: American Psychiatric Association; 2000.

25. Nolen-Hoeksema S, Morrow J. A prospective study of depression and posttraumatic stress symptoms after a natural disaster: the 1989 Loma Prieta Earthquake. J Pers Soc Psychol. 1991;61(1):115-121.

26. Treynor W, Gonzalez R, Nolen-Hoeksema S. Rumination reconsidered: a psychometric analysis. Cognit Ther Res. 2003;27(3):247-259.

27. Caselli G, Gemelli A, Querci S, et al. The effect of rumination on craving across the continuum of drinking behavior. Addict Behav. 2013; 38(12):2879-2883.

28. Flannery BA, Volpicelli JR, Pettinati HM. Psychometric properties of the Penn Alcohol Craving Scale. Alcohol Clin Exp Res. 1999;23(8): 1289-1295. 
29. Michielin P, Vidotto G, Altoè G, et al. Proposta di un nuovo strumento per la verifica dell'efficacia nella pratica dei trattamenti psicologici e psicoterapeutici [New questionnaire to evaluate psychological treatment effects]. G Ital Med Lav Ergon. 2008; 30(1 suppl A):A98-A104. Italian.

30. Bertolotti G, Michielin P, Vidotto G, et al. Metric qualities of the cognitive behavioral assessment for outcome evaluation to estimate psychological treatment effects. Neuropsychiatr Dis Treat. 2015;11:2449-2460.

31. Cohen J. Statistical Power Analysis for the Behavioral Sciences. New Jersey: LEA Publishers; 1988.

32. Sawilowsky S. New effect size rules of thumb. JMod Appl Stat Methods. 2009;8(2):467-474.

33. Cooney NL, Litt MD, Morse PA, Bauer LO, Gaupp L. Alcohol cue reactivity, negative mood reactivity, and relapse in treated alcoholic men. J Abnorm Psychol. 1997;106(2):243-250.

34. Levenson RW, Sher KJ, Grossman LM, Newman J, Newlin DB. Alcohol and stress response dampening: pharmacological effects, expectancy, and tension reduction. J Abnorm Psychol. 1980;89(4):528-538.

35. Agrawal A, Wetherill L, Bucholz KK, et al. Genetic influences on craving for alcohol. Addict Behav. 2013;38(2):1501-1508.

36. Spada MM, Wells A. Metacognitions about alcohol use in problem drinkers. Clin Psychol Psychother. 2006;13(2):138-143.

37. Zambon A, Soares Pinto SP, Agostini D, et al. Alcohol-addiction inpatient: characteristics of patients and rehabilitation program. Minerva Med. 2013;104(2):193-206. Italian.

38. Gorwood P. Alcohol dependence and anxious disorders: dangerous liaisons. Rev Prat. 2010;60(6):801-806. French.

39. Andersohn F, Kiefer F. Depressive mood and craving during alcohol withdrawal: association and interaction. Ger J Psychiatr. 2004;7(2):6-11.

40. Travaglini R, Giardinelli L. Alcoholism and sex difference. G Ital Psicopatol. 2005;11(4):437-444. Italian.

41. Yoo K-B, Park E-C, Jang S-Y, et al. Association between employment status change and depression in Korean adults. BMJ Open. 2016;6(3):e008570.
42. Malcolm R, Roberts JS, Wang W, Myrick H, Anton RF. Multiple previous detoxifications are associated with less responsive treatment and heavier drinking during an index outpatient detoxification. Alcohol. 2000;22(3):159-164.

43. Hertling I, Ramskogler K, Dvorak A, et al. Craving and other characteristics of the comorbidity of alcohol and nicotine dependence. Eur Psychiatry. 2005;20(5-6):442-450.

44. Tavares H, Zilberman ML, Hodgins DC, El-Guebaly N. Comparison of craving between pathological gamblers and alcoholics. Alcohol Clin Exp Res. 2005;29(8):1427-1431.

45. Fiabane E, Ottonello M, Zavan V, Pistarini C, Giorgi I. Motivation to change and posttreatment temptation to drink: a multicenter study among alcohol-dependent patients. Neuropsychiatr Dis Treat. 2017;3(13): 2497-2504.

46. Nolen-Hoeksema S. The role of rumination in depressive disorders and mixed anxiety/depressive symptoms. J Abnorm Psychol. 2000;109(3):504-511.

47. Hasegawa A. Translation and initial validation of the Japanese version of the Ruminative Responses Scale. Psychol Rep. 2013;112(3): 716-726.

48. Watkins ER. Depressive rumination: investigating mechanisms to improve cognitive behavioural treatments. Cogn Behav Ther. 2009;38(1):8-14.

49. Watkins E, Teasdale JD. Adaptive and maladaptive self-focus in depression. J Affect Disord. 2004;82(1):1-8.

50. Joormann J, Dkane M, Gotlib IH. Adaptive and maladaptive components of rumination? Diagnostic specificity and relation to depressive biases. Behav Ther. 2006;37(3):269-280.

51. Bottlender M, Soyka M. Impact of craving on alcohol relapse during, and 12 months following, outpatient treatment. Alcohol Alcohol. 2004;39(4):357-361.

52. Breiner MJ, Stritzke WGK, Lang AR. Approaching avoidance. A step essential to the understanding of craving. Alcohol Res Health. 1999; 23(3):197-206.
Neuropsychiatric Disease and Treatment

\section{Publish your work in this journal}

Neuropsychiatric Disease and Treatment is an international, peerreviewed journal of clinical therapeutics and pharmacology focusing on concise rapid reporting of clinical or pre-clinical studies on a range of neuropsychiatric and neurological disorders. This journal is indexed on PubMed Central, the 'PsycINFO' database and CAS,

\section{Dovepress}

and is the official journal of The International Neuropsychiatric Association (INA). The manuscript management system is completely online and includes a very quick and fair peer-review system, which is all easy to use. Visit http://www.dovepress.com/testimonials.php to read real quotes from published authors. 